\title{
Perivascular fatty tissue at the brachial artery is linked to insulin resistance but not to local endothelial dysfunction
}

\author{
K. Rittig • K. Staib • J. Machann • M. Böttcher • \\ A. Peter - F. Schick • C. Claussen • N. Stefan • \\ A. Fritsche $\cdot$ H.-U. Häring $•$ B. Balletshofer
}

Received: 23 April 2008 / Accepted: 14 July 2008 / Published online: 20 August 2008

(C) Springer-Verlag 2008

\begin{abstract}
Aims/hypothesis Different ectopic fat depots, such as visceral or hepatic fat, are known to affect whole body insulin sensitivity. It has recently been hypothesised that differences in perivascular adipose tissue (PVAT) mass around resistance vessels may also contribute to insulin resistance, possibly via direct vascular effects leading to reduced capillary cross-sectional area in the muscle, which in turn affects muscular blood flow and glucose uptake. Based on this, the aim of the present study was to test whether PVAT around conduit arteries (i.e. the brachial artery) influences NO bioavailability, expressed as flowmediated dilation (FMD), or insulin sensitivity in humans in vivo.

Methods Insulin sensitivity was measured by OGTT in all 95 participants (59 women, 36 men; median age 47 years, range 19-66 years) and by the gold standard, a euglycaemic-hyperinsulinaemic clamp, in a randomly selected subgroup of 33 participants. Quantification of the different fat compartments, including PVAT around the brachial artery, was achieved by high-resolution magnetic resonance imaging $(1.5 \mathrm{~T})$. Blood flow and FMD were measured at
\end{abstract}

K. Rittig $\cdot$ K. Staib $\cdot$ A. Peter $\cdot$ N. Stefan $\cdot$ A. Fritsche $\cdot$

H.-U. Häring $•$ B. Balletshofer $(\bowtie)$

Department of Endocrinology, Diabetes, Vascular Medicine,

Nephrology and Clinical Chemistry, University of Tübingen,

Otfried-Müller Straße 10,

72076 Tübingen, Germany

e-mail: bernd.balletshofer@med.uni-tuebingen.de

J. Machann • M. Böttcher $\cdot$ F. Schick $\cdot$ C. Claussen

Section of Experimental Radiology,

Department of Diagnostic and Interventional Radiology,

University of Tübingen,

Tübingen, Germany the brachial artery using high-resolution $(13 \mathrm{MHz})$ ultrasound, after $5 \mathrm{~min}$ of forearm occlusion.

Results PVAT was negatively correlated with insulin sensitivity and the post-ischaemic increase in blood flow. The association between PVAT and insulin sensitivity $(r=$ $-0.54, \beta=-0.37, p=0.009$ ) was independent of age, sex, visceral adipose tissue, liver fat, BMI and further cardiovascular risk factors. No correlation could be detected between PVAT and local endothelial function. However, we observed an independent association between PVAT and post-ischaemic increase in blood flow $(r=-0.241 ; \beta=-1.69$; $p=0.02$ ).

Conclusions/interpretation PVAT seems to play an independent role in the pathogenesis of insulin resistance. This may be due to direct vascular effects influencing muscular blood flow.

Keywords Endothelial dysfunction · Insulin resistance .

Perivascular fat

\begin{tabular}{ll}
\multicolumn{2}{l}{ Abbreviations } \\
FMD & flow-mediated dilation \\
GTN & glyceryl trinitrate \\
hsCRP & highly sensitive C-reactive protein \\
ISI & insulin sensitivity index \\
LF & liver fat \\
PI3-kinase & phosphatidylinositol 3-kinase \\
PVAT & perivascular adipose tissue \\
VAT & visceral adipose tissue
\end{tabular}

Introduction

Insulin resistance, a key component of the metabolic syndrome, is strongly associated with obesity. It is well 
established that this association is dependent on the localisation and endocrine activity of the different fat stores of the body. In particular, ectopic fat stores such as liver fat (LF) and visceral adipose tissue (VAT) show a significant association with whole body insulin sensitivity [1].

Fatty tissue is believed to influence insulin sensitivity through the secretion of certain cyto- and chemokines ('adipokines') that interact directly or indirectly with the insulin signalling pathway [2-4].

Another mechanism by which insulin sensitivity is thought to be negatively influenced is reduced blood flow in the muscle, one of the main target organs for glucose uptake. According to the so-called 'capillary recruitment theory' [5], reduced capillary cross-sectional area in the muscle leads to impaired glucose supply to muscle cells and, subsequently, to reduced insulin-mediated glucose uptake. This proposed mechanism partly explains the link between insulin resistance and circulation.

Based on growing evidence that it is not obesity per se that is important for the development of insulin resistance, but, rather, where the fat is localised [6], it has been hypothesised that [7] perivascular fat (PVAT) around resistance vessels constitutes an ectopic fat compartment that may be relevant to the pathogenesis of insulin resistance. According to this hypothesis, PVAT around arterioles secretes adipocytokines that inhibit the insulin-mediated signalling cascade via phosphatidylinositol 3-kinase (PI3-K) in the endothelial cell [8], resulting in insulin resistance. Simultaneously, the Akt pathway is inhibited, leading to reduced endothelial nitric oxide synthase activity [9, 10], which attenuates NO bioavailability.

Based on these observations, the aim of the present study was to determine whether PVAT around conduit arteries (i.e. the brachial artery) influences NO bioavailability, expressed as flow-mediated dilation (FMD), or insulin sensitivity in humans in vivo. We therefore measured PVAT around the brachial artery and tested its associations with insulin resistance and with local endothelial dysfunction.

\section{Methods}

\section{Study population}

We studied a total of 95 participants (59 women, 36 men; median age 47 years, range 19-66 years). The examinations were performed in individuals participating in the ongoing Tuebingen Lifestyle Intervention Program (TULIP; EudraCT registration no. 2008-000442-31) study, in which individuals at increased risk of developing type 2 diabetes are metabolically screened and several vascular variables are determined [11]. The individuals have one or more of the following diabetes risk factors: being overweight (BMI $\left.>27 \mathrm{~kg} / \mathrm{m}^{2}\right)(n=53)$, being a first-degree relative of a patient with type 2 diabetes $(n=78)$ or having impaired glucose tolerance $(n=20)$.

All 95 individuals underwent an OGTT, and a randomly selected subgroup of 33 individuals ( 21 women, 12 men; median age 50 years, range $22-63$ years) also underwent a euglycaemic-hyperinsulinaemic clamp. Informed written consent was given by each participant. The study was approved by the ethics committee of the University of Tübingen, Germany.

\section{OGTT}

A standardised OGTT was performed using a solution containing $75 \mathrm{~g}$ glucose at 08:00 hours, after an overnight fast of $12 \mathrm{~h}$. An insulin sensitivity index (ISI) was calculated according to the criteria described by Matsuda and DeFronzo [12], referred to throughout the remainder of this article as ISI-OGTT.

\section{Euglycaemic-hyperinsulinaemic clamp}

After participants had undergone a $12 \mathrm{~h}$ overnight fast, an antecubital vein was cannulated for infusion of insulin and glucose. A dorsal hand vein of the contralateral arm was cannulated and placed under a heating device to permit sampling of arterialised blood. After a basal blood sample had been drawn, participants received a primed insulin infusion at a rate of $1.0 \mathrm{mU} \mathrm{kg}^{-1} \mathrm{~min}^{-1}$ for $2 \mathrm{~h}$. A blood sample was drawn every 5 min for determination of blood glucose, and the glucose infusion was adjusted to maintain the fasting glucose level. An insulin sensitivity index (in $\mu \mathrm{mol} \mathrm{kg}{ }^{-1}$ $\min ^{-1}[\mathrm{pmol} / 1]^{-1}$ ) for systemic glucose uptake was calculated as the mean infusion rate of glucose (in $\mu \mathrm{mol} \mathrm{kg} \mathrm{kg}^{-1}$ $\min ^{-1}$ ) necessary to maintain euglycaemia during the last $60 \mathrm{~min}$ of the euglycaemic-hyperinsulinaemic clamp, divided by the steady-state serum insulin concentration (in $\mathrm{pmol} / \mathrm{l})$. This is referred to throughout the remainder of this article as ISI-clamp.

Vascular variables

We used a non-invasive method to evaluate blood flow volume and FMD, described in 1992 by Celermajer et al. [13]. This method uses post-ischaemic (forearm) vasodilation, causing enhanced flow in the proximal (brachial) artery, which is regarded to result in the shear stressinduced release of $\mathrm{NO}$, and subsequent vasodilation of the brachial artery. FMD at the radial artery has been proved to be endothelium-dependent, since it can be blocked by $N$ monomethyl-L-arginine, an inhibitor of endothelial nitric oxide synthase [14]. We examined endothelium-dependent vasodilation using high-resolution $(13 \mathrm{MHz})$ ultrasound 
(AU5 Idea; Esaote Biomedica, Genoa, Italy) with an integrated ECG package. Participants fasted and refrained from smoking for at least $10 \mathrm{~h}$ prior to examination. The diameter of the brachial artery was measured from twodimensional ultrasound images created using a $13 \mathrm{MHz}$ linear-array transducer. The brachial artery was scanned in longitudinal sections, $2-10 \mathrm{~cm}$ proximal to the elbow. Lumen diameter was assessed according to the sonomorphological definition of Wendelhag et al. [15]. Participants rested for at least $10 \mathrm{~min}$ before the first baseline scans were recorded. Before compression, the mean blood flow velocity and the arterial diameter were measured. The blood flow velocity was measured continuously and the mean blood flow velocity was used to estimate blood flow volume. The formula used to calculate blood flow volume was $\pi \times(\text { artery diameter })^{2} \times$ blood flow velocity in $\mathrm{cm} / \mathrm{s}$.

Increased flow was induced by deflating a pneumatic tourniquet after a $5 \mathrm{~min}$ suprasystolic $(230 \mathrm{mmHg})$ forearm compression. The post-ischaemic blood flow volume was assessed immediately after cuff deflation and is reported as fold increase from baseline. Post-ischaemic diameter was measured 45-60 s after cuff deflation. The CV in blood flow measurement was $8.76 \%$. To exclude possible subendothelial alterations that might mimic endothelial dysfunction, vascular relaxation was also tested 4 min after sublingual administration of $0.4 \mu \mathrm{g}$ glyceryl trinitrate (GTN), an endothelial-independent vasodilator. The time span between the post-ischaemic and the pre-GTN scans had to be at least $15 \mathrm{~min}$, to allow vessel recovery. Vessel diameter was analysed using electronic calipers on frozen images over a length of the artery of at least $1 \mathrm{~cm}$. Three measurements were taken at each scan for three cardiac cycles at the end of the diastole (incident with the $\mathrm{R}$ wave on the ECG), and the mean was then calculated.

\section{MR examinations}

All MR examinations were performed in the early morning, after an overnight fast, on a $1.5 \mathrm{~T}$ whole body imager (Magnetom Sonata; Siemens Healthcare, Erlangen Germany).

MRI quantification of PVAT in the upper arm The amount of PVAT in the upper arm was determined by applying a T1-weighted gradient-echo sequence with following measurement variables: echo time, $4.8 \mathrm{~ms}$; repetition time, $91 \mathrm{~ms}$; slice thickness, $10 \mathrm{~mm}$; in-plane resolution, $0.8 \mathrm{~mm}$; seven slices; acquisition time, 1:18 min. The body coil was used as a receiver. Volunteers lay in a prone position with extended arms. The central slice was positioned $15 \mathrm{~cm}$ distal to the head of the humerus. The amount of PVAT in $\mathrm{cm}^{2}$ was quantified using the scanner software by manually drawing the contours of the fatty region between subcuta- neous fat and the brachial artery in all seven images, as shown in Fig. 1 for a single slice. After all seven slices had been measured, the mean (in $\mathrm{cm}^{2}$ ) was calculated. Testing of the reliability of the method by repeated measurements in five volunteers after repositioning in the scanner revealed intra-individual variations of below $10 \%$. Additionally, five datasets were evaluated by two observers (J. Machann and M. Böttcher), and these showed inter-observer differences of below $8 \%$.

MRI quantification of VAT Visceral adipose tissue was measured with an axial T1-weighted fast spin echo technique with a $1.5 \mathrm{~T}$ whole body imager (Magnetom Sonata) as described previously [16].

Magnetic resonance spectroscopy of the liver The amount of lipids in the liver was measured by localised ${ }^{1} \mathrm{H}$ magnetic resonance spectroscopy from a voxel within the posterior part of segment seven of the liver as described previously [17].

Measurement of highly sensitive C-reactive protein

Plasma highly-sensitive C-reactive protein (hsCRP) was measured using a latex-enhanced immunoturbidimetric method (Wide Range C-Reactive Protein kit) with the ADVIA 1650 Clinical Chemistry Analyzer (Siemens Medical Solutions Diagnostics, Fernwald, Germany)
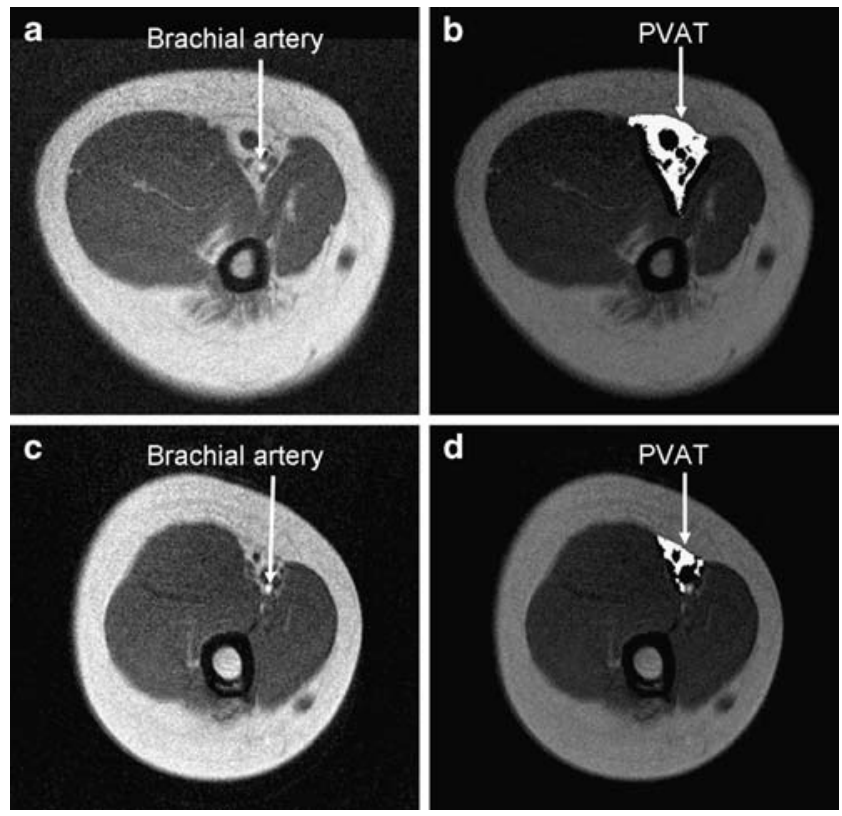

Fig. 1 T1-weighted MR images of the upper arm of two volunteers with high (a) and low (c) amounts of PVAT. Segmented PVAT is highlighted in (b) and (d) 
Statistical analyses

Data are provided as means \pm SD when values are distributed normally, and as median (range) when not normally distributed. Statistical comparison of phenotypes was performed using ANOVA for normally distributed variables. Non-normally distributed variables were log transformed to achieve normal distribution. If log transformation did not result in normal distribution, original data were used in a non-parametric test. The $\chi^{2}$ test was used when analysing differences between categorical variables such as sex or smoking. Multivariate linear regression analyses were performed to adjust for the effects of relevant covariates. A $p$ value of less than 0.05 was considered to be statistically significant. The analyses were conducted using the JMP statistical software package 4.0.4 (SAS Institute, Cary, NC, USA).

\section{Results}

\section{Anthropometrical and laboratory findings}

The clinical characteristics of the study population are shown in Table 1. The middle-aged study group was normotensive and slightly overweight. The glucose levels at baseline and at $2 \mathrm{~h}$ during the OGTT were $5.25 \pm 0.53$ and $6.99 \pm 2.05 \mathrm{mmol}$, respectively. As one part of the insulin resistance syndrome and cardiovascular risk profile, lipoprotein status, including total, HDL- and LDL-cholesterol and triacylglycerol, was normal, with exception of a moderately increased total cholesterol level of $5.27 \pm$ $1.08 \mathrm{mmol} / \mathrm{l}$.

Table 1 Demographics, anthropometrics and laboratory findings of the study population

\begin{tabular}{ll}
\hline Variable & All participants $(n=95)$ \\
\hline Age (years) & $47(19-66)$ \\
Sex (women $/ \mathrm{men})$ & $59 / 36$ \\
Systolic BP $(\mathrm{mmHg})$ & $125(90-180)$ \\
Diastolic BP $(\mathrm{mmHg})$ & $80(60-100)$ \\
BMI $\left(\mathrm{kg} / \mathrm{m}^{2}\right)$ & $27.7 \pm 4.9$ \\
Body fat $(\%)$ & $30.3 \pm 7.7$ \\
ISI-OGTT & $11.1(3.5-43.9)$ \\
ISI-clamp ${ }^{\text {a }}\left(\mu \mathrm{mol} \mathrm{kg}{ }^{-1} \mathrm{~min}^{-1}\right)$ & $0.06(0.02-0.13)$ \\
Triacylglycerol $(\mathrm{mmol} / \mathrm{l})$ & $1.33(0.42-18.3)$ \\
Total cholesterol $(\mathrm{mmol} / \mathrm{l})$ & $5.27 \pm 1.08$ \\
HDL-cholesterol $(\mathrm{mmol} / \mathrm{l})$ & $1.35(0.25-2.65)$ \\
LDL-cholesterol $(\mathrm{mmol} / \mathrm{l})$ & $3.09(0.53-5.51)$ \\
\hline
\end{tabular}

Data are mean $\pm \mathrm{SD}$ when values are distributed normally, and median (range) when not normally distributed

${ }^{\mathrm{a}} n=33$
Vascular variables

Mean basal brachial artery diameter, measured by highresolution ultrasound, was $3.67 \pm 0.76 \mathrm{~mm}$ (Table 2). As expected, there was a significant difference between sexes (women vs men: $3.2 \pm 0.4$ vs $4.39 \pm 0.6 \mathrm{~mm}, p<0.0001$ ). FMD was $6.37 \%(0.73-26.24 \%)$ in the whole study group.

Vasodilation 4 min after sublingual application of $0.4 \mu \mathrm{g}$ GTN was $16.41 \%$ (1.57-37.48\%). All participants showed vasodilation after GTN to a different extent; therefore, subendothelial alterations mimicking endothelial dysfunction can be ruled out in our study population.

Adipose tissue measurement

The median amount of PVAT was $1.02(0.38-3.46) \mathrm{cm}^{2}$. We observed positive correlations between PVAT and VAT $(r=$ $0.49, \beta=0.96, p<0.0001)$, PVAT and LF $(r=0.46, \beta=1.15$, $p<0.0001)$ and VAT and LF $(r=0.47, \beta=0.61, p<0.0001)$.

The subgroup of participants who underwent a glucose clamp examination ( $n=33)$ did not differ significantly from those who underwent the OGTT alone $(n=62)$ in terms of the above-mentioned variables, with the exception of a slightly lower FMD in the clamp subgroup (4.27\% [1.88-18.15\%] vs $7.55 \%[1.88-26.24 \%], p=0.02)$.

Fat distribution and insulin sensitivity

Although the different fat compartments were related to each other, we found distinct differences concerning their influence on insulin sensitivity. PVAT showed a strong negative correlation to the ISI-OGTT in univariate analysis ( $r=-0.42, \beta=-0.543, p<0.0001)$. After adjustment for age and sex, the correlation did not change considerably $(r=-0.41, \beta=-0.550, p<0.0001)$. As expected, there was also a negative correlation between VAT and the ISI-OGTT

Table 2 Vascular variables and fat compartments of the study population

\begin{tabular}{ll}
\hline Variable & All participants $(n=95)$ \\
\hline Basal brachial artery diameter $(\mathrm{mm})$ & $3.67 \pm 0.76$ \\
Arterial blood flow at baseline $(\mathrm{ml} / \mathrm{s})$ & $1.80(0.42-7.46)$ \\
Post-ischaemic blood flow volume & $4.56(2.35-13.19)$ \\
$\quad$ (fold increase from baseline) & \\
Flow-mediated dilation $(\%)$ & $6.37(0.73-26.24)$ \\
GTN-induced dilation $(\%)$ & $16.41(1.57-37.48)$ \\
Visceral adipose tissue & $2.07(0.18-6.44)$ \\
$\quad(\%$ body weight) & $1.98(0.1-22.6)$ \\
Liver fat $(\%)$ & $1.02(0.38-3.46)$ \\
Perivascular fat $\left(\mathrm{cm}^{2}\right)$ &
\end{tabular}

Data are mean \pm SD when values are distributed normally, and median (range) when not normally distributed 
$(r=-0.40, \beta=-0.263, p<0.0001)$. However, when age, sex, VAT, LF and other cardiovascular risk factors (systolic and diastolic blood pressure, total and LDL-cholesterol, BMI) were included in one multivariate model, the relationship between PVAT and the ISI-OGTT was no longer significant.

In the subgroup that underwent a euglycaemic-hyperinsulinaemic clamp $(n=33)$, the univariate effect of PVAT on insulin sensitivity was even stronger $(r=-0.65, \beta=$ $-0.724, p<0.0001)$. Adjustment for age and sex did not change this association considerably $(r=-0.621, \beta=-0.684$, $p=0.0002)$. VAT again showed a negative association with the ISI-clamp in univariate analysis $(r=-0.54, \beta=-0.306$, $p=0.001)$. Adjustment for age, sex, PVAT, VAT, LF and other cardiovascular risk factors (systolic and diastolic blood pressure, total and LDL-cholesterol, BMI) in one statistical model resulted in a persistent, strong negative association between PVAT and the ISI-clamp (Table 3, Fig. 2).

Fat distribution and vascular variables

The fat compartments studied did not significantly influence local FMD at the brachial artery either in univariate analysis or in the multivariate model adjusted for age, sex, basal artery diameter and post-ischaemic mean blood flow velocity.

GTN-induced vasodilation was not affected by PVAT, ruling out subendothelial alterations as an explanation for the above-mentioned observation. However, PVAT was negatively correlated with post-ischaemic blood flow
Table 3 Factors influencing clamp-derived ISI

\begin{tabular}{|c|c|c|c|c|}
\hline Model & Variable & Estimate $(\beta)$ & $\mathrm{SE}$ & $p$ value \\
\hline \multirow[t]{8}{*}{ Model 1} & Sex & 0.005 & 0.004 & 0.19 \\
\hline & Age & 0.0004 & 0.0003 & 0.29 \\
\hline & Systolic BP & -0.0002 & 0.0002 & 0.33 \\
\hline & Diastolic BP & 0.0001 & 0.0005 & 0.76 \\
\hline & Total cholesterol & 0.00002 & 0.004 & 0.99 \\
\hline & LDL-cholesterol & -0.008 & 0.0056 & 0.14 \\
\hline & CRP & -0.002 & 0.003 & 0.39 \\
\hline & PVAT & -0.045 & 0.01 & 0.0002 \\
\hline \multirow[t]{9}{*}{ Model 2} & Sex & 0.004 & 0.004 & 0.32 \\
\hline & Age & 0.0003 & 0.0003 & 0.42 \\
\hline & Systolic BP & -0.0002 & 0.0002 & 0.53 \\
\hline & Diastolic BP & 0.0002 & 0.0005 & 0.68 \\
\hline & Total cholesterol & -0.0009 & 0.004 & 0.81 \\
\hline & LDL-cholesterol & -0.005 & 0.006 & 0.36 \\
\hline & CRP & 0.0002 & 0.003 & 0.94 \\
\hline & BMI & -0.001 & 0.001 & 0.13 \\
\hline & PVAT & -0.035 & 0.011 & 0.007 \\
\hline \multirow[t]{10}{*}{ Model 3} & Sex & 0.01 & 0.006 & 0.1 \\
\hline & Age & 0.00005 & 0.0004 & 0.89 \\
\hline & Systolic BP & -0.00013 & 0.0003 & 0.65 \\
\hline & Diastolic BP & 0.00005 & 0.0004 & 0.92 \\
\hline & Total cholesterol & -0.002 & 0.004 & 0.61 \\
\hline & LDL-cholesterol & -0.006 & 0.006 & 0.33 \\
\hline & CRP & -0.0003 & 0.003 & 0.92 \\
\hline & BMI & -0.002 & 0.001 & 0.052 \\
\hline & VAT & 0.007 & 0.005 & 0.18 \\
\hline & PVAT & -0.038 & 0.12 & 0.004 \\
\hline \multirow[t]{11}{*}{ Model 4} & Sex & 0.01 & 0.006 & 0.07 \\
\hline & Age & 0.00006 & 0.0004 & 0.89 \\
\hline & Systolic BP & -0.0002 & 0.0003 & 0.50 \\
\hline & Diastolic BP & 0.0002 & 0.0005 & 0.75 \\
\hline & Total cholesterol & -0.0003 & 0.004 & 0.95 \\
\hline & LDL-cholesterol & -0.007 & 0.006 & 0.28 \\
\hline & CRP & -0.002 & 0.004 & 0.64 \\
\hline & BMI & -0.002 & 0.001 & 0.19 \\
\hline & VAT & 0.008 & 0.005 & 0.13 \\
\hline & $\mathrm{LF}$ & -0.006 & 0.005 & 0.27 \\
\hline & PVAT & -0.04 & 0.01 & 0.009 \\
\hline
\end{tabular}




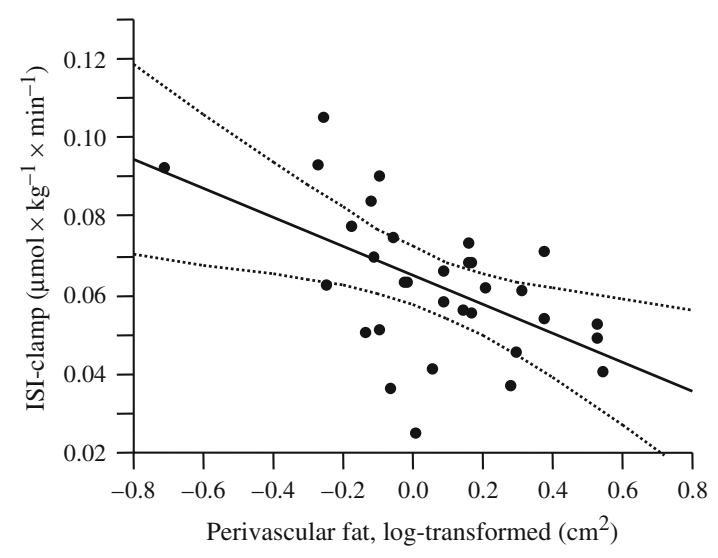

Fig. 2 Correlation between PVAT and insulin sensitivity, adjusted for age, sex, VAT, liver fat, BMI, CRP, systolic and diastolic BP and total and LDL-cholesterol $(r=-0.54, \beta=-0.04, p=0.009)$. The graph shows the regression line (solid line) and the $95 \%$ CI (dotted lines)

volume at the brachial artery $(r=-0.37, \beta=-2.25, p=$ $0.0003)$. In multivariate analysis, this association was independent of potentially confounding variables such as age, sex and VAT $(r=-0.24, \beta=-1.69, p=0.02)$. On the other hand, when adjusted for age and sex, VAT and LF were not associated with post-ischaemic blood flow volume.

Fat distribution and hsCRP levels

In our population, median serum levels of hsCRP were 1.1 (0.1-27.2) $\mathrm{mg} / \mathrm{l}$. We observed a weak, but significant positive correlation between hsCRP serum levels and PVAT, independently of age, sex, VAT and even LF $(r=0.28, \beta=0.92, p=0.009)$.

\section{Discussion}

We observed a close relationship between PVAT determined at the brachial artery and insulin sensitivity in humans in vivo. Although there were correlations between the amounts of fat in each compartment, PVAT is not merely an epiphenomenon of adiposity. As expected, VAT and LF were also associated with insulin sensitivity in univariate analysis (data not shown). However, in multivariate models that took into account age, sex, VAT, LF, several cardiovascular risk factors and PVAT, PVAT was shown to be independently associated with insulin sensitivity. In our study population, this association could be detected in the subgroup randomly selected to undergo a euglycaemic-hyperinsulinaemic clamp (ISI-clamp), the gold standard for insulin-sensitivity measurements. There was no independent association between PVAT and the ISI-OGTT. This finding may be explained by the fact that the ISI clamp is considered to reflect muscular insulin resistance to a greater extent, and PVAT around the brachial artery is more likely to influence insulin resistance in this area, whereas the ISI-OGTT represents whole body insulin resistance, and is influenced by several other variables, such as liver fat. Thus, our results suggest a relevant effect of PVAT in the regulation of insulin sensitivity. According to a currently discussed hypothesis, PVAT may influence insulin sensitivity via inhibition of the insulin signal cascade, via paracrine and 'vasocrine' signalling [7]. Secretion of IL-6 or TNF leads to inhibition of PI3-kinase, resulting in insulin resistance [8]. This inhibitory effect has been demonstrated in vitro as well as in vivo $[2,3,18]$. That PVAT releases proinflammatory cytokines has recently been shown in vitro by Henrichot et al. [19].

It should be emphasised that the hypothesis put forward by Yudkin et al. [7] explicitly concerns peri-arteriolar fat in muscle. Our study investigated perivascular fat around conduit arteries. Therefore, the mechanism by which PVAT around the brachial artery influences insulin sensitivity on a molecular level needs to be clarified in further studies.

Another consequence of PI3-kinase inhibition and subsequent downstream inhibition of Akt is an inhibitory effect on the endothelial NO synthase pathway [9, 10]. Reduced NO bioavailability could therefore cause endothelial dysfunction. To test whether this effect can be observed in conduit arteries in vivo, we measured local NO bioavailability at the brachial artery, expressed as FMD. Interestingly, we could not detect an influence of PVAT on FMD at the brachial artery, excluding a direct effect of PVAT on local NO bioavailability in this vascular segment. Thus, the hypothesis that PVAT also influences vascular function is not applicable to conduit arteries. We observed a weak, but independently significant negative association between PVAT and the post-ischaemic increase in flow volume at the brachial artery. The experimental post-ischaemic increase in blood flow volume, as induced in this study, is mediated by forearm vasodilation and therefore by the distal vascular cross-sectional area, probably reflecting microvascular capacity. According to the capillary recruitment theory [5], reduced blood flow as a result of a reduced capillary cross-sectional area may be one mechanism that contributes to insulin resistance $[5,20]$. In previous studies, muscle perfusion has been shown to modulate insulinmediated glucose uptake [20-22], which is reduced in the insulin-resistant state [23, 24].

Subclinical inflammation is known to be associated with insulin resistance, as well as endothelial dysfunction. Since serum levels of IL-6 and TNF do not represent tissue levels, we used hsCRP as a surrogate variable for subclinical inflammation. In our population we detected a positive association between PVAT and serum hsCRP levels, indicating that individuals with higher amounts of PVAT suffer from a potentially higher inflammatory burden, 
which is known to be a risk factor for diabetes as well as a cardiovascular risk factor [25].

A potential limitation of our study is the heterogeneity of the study group. Metabolically, a lean first-degree relative with a genetic background of diabetes cannot simply be compared with an overweight insulin-resistant individual. It is evident that our conclusions, which are based on heterogeneous phenotypes, may not be applicable to all phenotypes.

In conclusion, PVAT seems to represent a novel and important adipose tissue compartment for the regulation of insulin sensitivity, independent of other fat compartments. The underlying mechanisms by which PVAT regulates insulin sensitivity may in part be due to differences in muscular blood flow. However, local endothelial function of conduit arteries is not affected by PVAT.

Acknowledgements This study was supported by a research grant from the German research foundation (Deutsche Forschungsgemeinschaft, grant no. DFG-KFO 114/1)

Duality of interest The authors declare that there is no duality of interest associated with this manuscript.

\section{References}

1. Despres JP, Lemieux I (2006) Abdominal obesity and metabolic syndrome. Nature 444:881-887

2. Mohamed F, Monge JC, Gordon A et al (1995) Lack of role for nitric oxide (NO) in the selective destabilization of endothelial $\mathrm{NO}$ synthase mRNA by tumor necrosis factor-alpha. Arterioscler Thromb Vasc Biol 15:52-57

3. Zhang L, Wheatley CM, Richards SM et al (2003) TNF-alpha acutely inhibits vascular effects of physiological but not high insulin or contraction. Am J Physiol Endocrinol Metab 285:E654-E660

4. Hotamisligil GS (1999) The role of TNFalpha and TNF receptors in obesity and insulin resistance. J Intern Med 245:621-625

5. Baron AD, Tarshoby M, Hook G et al (2000) Interaction between insulin sensitivity and muscle perfusion on glucose uptake in human skeletal muscle: evidence for capillary recruitment. Diabetes 49:768-774

6. Powell K (2007) Obesity: the two faces of fat. Nature 447:525527

7. Yudkin JS, Eringa E, Stehouwer CD (2005) 'Vasocrine' signalling from perivascular fat: a mechanism linking insulin resistance to vascular disease. Lancet 365:1817-1820

8. Khan AH, Pessin JE (2002) Insulin regulation of glucose uptake: a complex interplay of intracellular signalling pathways. Diabetologia 45:1475-1483
9. Eringa EC, Stehouwer CD, Nieuw Amerongen GP et al (2004) Vasoconstrictor effects of insulin in skeletal muscle arterioles are mediated by ERK1/2 activation in endothelium. Am J Physiol Heart Circ Physiol 287:H2043-H2048

10. Rattigan S, Clark MG, Barrett EJ (1997) Hemodynamic actions of insulin in rat skeletal muscle: evidence for capillary recruitment. Diabetes 46:1381-1388

11. Schafer S, Kantartzis K, Machann J et al (2007) Lifestyle intervention in individuals with normal versus impaired glucose tolerance. Eur J Clin Invest 37:535-543

12. Matsuda M, DeFronzo RA (1999) Insulin sensitivity indices obtained from oral glucose tolerance testing: comparison with the euglycemic insulin clamp. Diabetes Care 22:1462-1470

13. Celermajer DS, Sorensen KE, Georgakopoulos D et al (1993) Cigarette smoking is associated with dose-related and potentially reversible impairment of endothelium-dependent dilation in healthy young adults. Circulation 88:2149-2155

14. Joannides R, Haefeli WE, Linder L et al (1995) Nitric oxide is responsible for flow-dependent dilatation of human peripheral conduit arteries in vivo. Circulation 91:1314-1319

15. Wendelhag I, Gustavsson T, Suurkula M, Berglund G, Wikstrand J (1991) Ultrasound measurement of wall thickness in the carotid artery: fundamental principles and description of a computerized analysing system. Clin Physiol 11:565-577

16. Machann J, Thamer C, Schnoedt B et al (2005) Standardized assessment of whole body adipose tissue topography by MRI. J Magn Reson Imaging 21:455-462

17. Stefan N, Machicao F, Staiger H et al (2005) Polymorphisms in the gene encoding adiponectin receptor 1 are associated with insulin resistance and high liver fat. Diabetologia 48:2282-2291

18. Hotamisligil GS (2003) Inflammatory pathways and insulin action. Int J Obes Relat Metab Disord 27(Suppl 3):S53-S55

19. Henrichot E, Juge-Aubry CE, Pernin A et al (2005) Production of chemokines by perivascular adipose tissue: a role in the pathogenesis of atherosclerosis? Arterioscler Thromb Vasc Biol 25:2594-2599

20. Baron AD, Steinberg HO, Chaker $H$ et al (1995) Insulinmediated skeletal muscle vasodilation contributes to both insulin sensitivity and responsiveness in lean humans. J Clin Invest 96 : 786-792

21. Clark MG, Colquhoun EQ, Rattigan S et al (1995) Vascular and endocrine control of muscle metabolism. Am J Physiol 268:E797E812

22. Hespel P, Vergauwen L, Vandenberghe K, Richter EA (1995) Important role of insulin and flow in stimulating glucose uptake in contracting skeletal muscle. Diabetes 44:210-215

23. Laakso M, Edelman SV, Brechtel G, Baron AD (1992) Impaired insulin-mediated skeletal muscle blood flow in patients with NIDDM. Diabetes 41:1076-1083

24. Vollenweider P, Randin D, Tappy L et al (1994) Impaired insulininduced sympathetic neural activation and vasodilation in skeletal muscle in obese humans. J Clin Invest 93:2365-2371

25. Balletshofer BM, Haap M, Rittig K et al (2005) Early carotid atherosclerosis in overweight nondiabetic individuals is associated with subclinical chronic inflammation independent of underlying insulin resistance. Horm Metab Res 37:331-335 